\title{
Surgical versus non-surgical interventions in patients with adolescent idiopathic scoliosis (Protocol)
}

Bettany-Saltikov J, Weiss HR, Chockalingam N, Taranu R, Srinivas S, Hogg J, Whittaker V, Kalyan RV
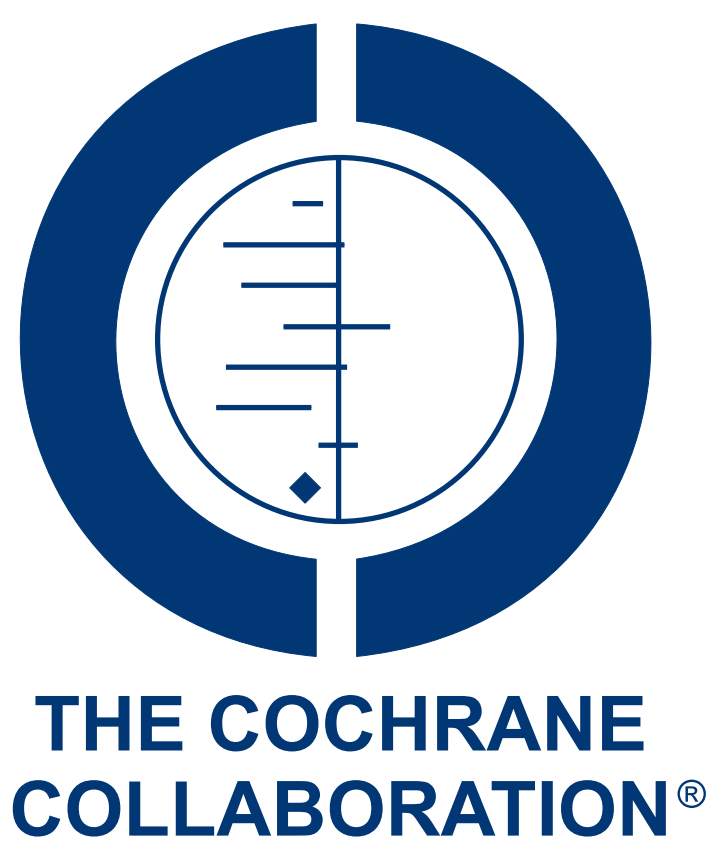

This is a reprint of a Cochrane protocol, prepared and maintained by The Cochrane Collaboration and published in The Cochrane Library 2013, Issue 7

http://www.thecochranelibrary.com

\section{WILEY}

Surgical versus non-surgical interventions in patients with adolescent idiopathic scoliosis (Protocol)

Copyright $\odot 2013$ The Cochrane Collaboration. Published by John Wiley \& Sons, Ltd. 
TABLE OF CONTENTS

HEADER . . . . . . . . . . . . . . . . . . . . . . . . . . . . . . . . . . . . 1

ABSTRACT . . . . . . . . . . . . . . . . . . . . . . . . . . . . . . . . . . . . . . 1

BACKGROUND . . . . . . . . . . . . . . . . . . . . . . . . . . . . . . . . . . . .

OBJECTIVES . . . . . . . . . . . . . . . . . . . . . . . . . . . . . . . . . . . . . . . 5

METHODS . . . . . . . . . . . . . . . . . . . . . . . . . . . . . . . . . . . . . . 5

ACKNOWLEDGEMENTS . . . . . . . . . . . . . . . . . . . . . . . . . . . . . . . . . . . .

REFERENCES . . . . . . . . . . . . . . . . . . . . . . . . . . . . . . . . . . . . . . 8

APPENDICES . . . . . . . . . . . . . . . . . . . . . . . . . . . . . . . . . . . . . . 12

CONTRIBUTIONS OF AUTHORS . . . . . . . . . . . . . . . . . . . . . . . . . . . . . . . . . . . 20

DECLARATIONS OF INTEREST . . . . . . . . . . . . . . . . . . . . . . . . . . . . . . . . . . 20 


\title{
[Intervention Protocol]
}

\section{Surgical versus non-surgical interventions in patients with adolescent idiopathic scoliosis}

\author{
Josette Bettany-Saltikov ${ }^{1}$, Hans-Rudolf Weiss ${ }^{2}$, Nachiappan Chockalingam ${ }^{3}$, Razvan Taranu ${ }^{4}$, Shreya Srinivas ${ }^{5}$, Julie Hogg ${ }^{1}$, Victoria \\ Whittaker $^{1}$, Raman V Kalyan ${ }^{6}$ \\ ${ }^{1}$ School of Health and Social Care, University of Teesside, Middlesbrough, UK. ${ }^{2}$ Spinal Deformities Rehabilitation Services, Ortho- \\ pedic Practice, Gensingen, Germany. ${ }^{3}$ Faculty of Health, Staffordshire University, Stoke-on-Trent, UK. ${ }^{4}$ Department of Trauma and \\ Orthopaedics, Northumbria Healthcare NHS Foundation Trust, Ashington, UK. ${ }^{5}$ Northern Deanery, James Cook University Hospital, \\ Middlesbrough, UK. ${ }^{6}$ James Cook University Hospital, Middlesbrough, UK
}

Contact address: Josette Bettany-Saltikov, School of Health and Social Care, University of Teesside, Victoria Road, Middlesbrough, Cleveland, TS13BA, UK. j.b.saltikov@tees.ac.uk.

Editorial group: Cochrane Back Group.

Publication status and date: New, published in Issue 7, 2013.

Citation: Bettany-Saltikov J, Weiss HR, Chockalingam N, Taranu R, Srinivas S, Hogg J, Whittaker V, Kalyan RV. Surgical versus non-surgical interventions in patients with adolescent idiopathic scoliosis. Cochrane Database of Systematic Reviews 2013, Issue 7. Art. No.: CD010663. DOI: 10.1002/14651858.CD010663.

Copyright (C) 2013 The Cochrane Collaboration. Published by John Wiley \& Sons, Ltd.

\begin{abstract}
A B S T R A C T
This is the protocol for a review and there is no abstract. The objectives are as follows:

The objective of this review is to evaluate and compare changes in trunk balance, progression of scoliosis, cosmetic issues, quality of life, disability, psychological issues and back pain, as well as adverse effects, with both surgical and non-surgical interventions provided in the short term (a few months) and in the long term (over 20 years).
\end{abstract}

\section{B A C K G R O U N D}

\section{Description of the condition}

Scoliosis is a complex three-dimensional deformity of the spine that comprises a lateral curvature in the frontal plane (this is a vertical plane that divides the body into front and back halves), thoracic lordosis in the sagittal plane (this is a vertical plane that divides the body into right and left halves) and a posterior rib hump, which is produced by rotation of the vertebrae in the transverse plane (horizontal plane); this results in the posterior elevation of the rib cage on the convex side of the curve and a depression on the concave side (Bradford 1987). These underlying skeletal changes are usually reflected by a change in back shape, the unsightly shape of which is generally more of a concern to the patient than is the underlying skeletal deformity (White 1990). The condition if left untreated results in altered spinal mechanics and degenerative changes that lead to pain, loss of spinal mobility and possible loss of function or disability. Cardiac and respiratory dysfunction may also accompany these symptoms, depending on the time of onset of the deformity (White 1990). These physical changes are accompanied by the psychological consequences resulting from the unsightly and deformed shape of the back: a restricted social life, a lower marriage rate, a higher divorce rate, fewer children per marriage and increased psychiatric consultations, including eating disorders and increased suicide rate, have all been reported (Freidel 
2002).

Whilst scoliosis may be the expression of a disease, it can also occur secondary to certain diseases and conditions that affect the nervous and muscular systems of the body. The deformity can be caused by defects in spine formation at the embryo stage, or it can be part of certain syndromes. Very rarely, scoliosis can occur secondary to tumours. However, most cases of scoliosis $(80 \%$ to 90\%) are called 'Idiopathic' because the underlying cause cannot be ascertained. Adolescent idiopathic scoliosis (AIS), which is the

most common diagnosis, usually develops during adolescence- a period of rapid growth (Kanayama 1996; Stokes 1996).

According to the Scoliosis Research Society and the International Society on Scoliosis Orthopaedic Rehabilitation and Treatment (SOSORT Guidelines 2012), the prevalence of AIS is 2\% to $3 \%$ in the general population. Almost $10 \%$ of patients with AIS will require some form of treatment, and up to $0.1 \%$ will eventually require surgery (Lonstein 2006). AIS is more commonly found in females (female/male ratio is around 7:1) and, except for extreme cases, AIS does not typically cause any health problems during growth; however, the resulting surface deformity frequently has a negative impact on adolescents that can give rise to quality of life issues and, in the worst cases, psychological disturbances (Reichel 2003).

The aetiology or causation of idiopathic scoliosis remains unclear (Sevastik 1997; Stokes 1997; Machida 1999; Burwell 2000). Many theories on the causes of scoliosis have been proposed, such as the neuromuscular, growth and genetic theories. White 1990 sums up all hypotheses related to the cause of scoliosis as follows: "The normal spine in a growing person has a precise, precarious, delicate mechanical balance. Asymmetrical changes in primary structures, support structures, growth centres, the position of the spine and related neural or muscular components can all result in the development of scoliosis."

The potential for curve progression has been shown to be related to several factors, including the patient's gender, age, curve magnitude, bone maturity, rate of growth and growth potential at presentation. Dickson 1984 demonstrated that when curves of 10 degrees Cobb and above were considered, the female-to-male ratio was 1.6:1. The Cobb angle is a method of measuring the angle of the spine that was devised by a surgeon named Cobb (Lonstein 2006). This value increased to $12: 1$ when curves greater than 20 degrees Cobb were considered. Female-to-male ratios for treatment were reported at 7:1 (Rogala 1978). Moreover, when combining curves of all magnitudes, Lonstein 1984 found a negative correlation of age with the percentage incidence of progression. This means that the younger the child at presentation, the greater is the likelihood of progression. The same negative correlation is shown with the Risser sign. This measures how much mature bone has developed (ossification) in the upper rim of the pelvis (iliac crest). The greater the maturity of the child, the greater is the Risser sign. A low Risser sign indicates that greater potential for growth is left, and consequently the potential for curve progression is greater (Bridwell 1999 and Lonstein 2006). Curve magnitude, however, was found to have a positive correlation with the percentage incidence of progression. Thus the greater the magnitude of the curve at presentation, the greater is the potential for progression.

Other factors taken into consideration when growth potential is determined are the changes in secondary sexual characteristics that take place during the growth spurt. Different results have been reported on the progression of various curve patterns. For example, Clarisse 1974 and Fustier 1980 reported that double curves progressed most in their studies, with an incidence of $67 \%$ and 75\%, respectively. Conversely, Bunnell 1986 and Lonstein 1984 reported that thoracic curves were most progressive. All authors, however, demonstrated that lumbar curves progressed least. Other parameters of prognostic value include apical vertebral rotation (Weinstein 1983; Perdriolle 1985) and the rib vertebral angle (Mehta 1972). When potential for curve progression is assessed, no single factor is taken in isolation, but all factors are taken into account in attempts to predict the likelihood for progression and make a treatment decision.

Depending on the age of the individual at diagnosis, scoliosis evolves and may deteriorate rapidly during periods of fast growth spurt (Goldberg 2002; Asher 2006; Hawes 2006b). Whilst children grow until they have fully matured, growth is more rapid (growth spurt) during certain periods of childhood and adolescence (Weiss 2012).

Early diagnosis is difficult, especially in countries where scoliosis school screening is not implemented, as this condition is most often painless. External change to the body shape is minimal in the early stages and most changes in back shape occur predominantly on the back of the trunk, which makes it difficult for patients to see, and it can be concealed by their clothing (Roaf 1980). Treatment of idiopathic scoliosis is determined by the deformity itself. As most patients with adolescent idiopathic scoliosis progress during growth, the main aims of all interventions are to limit or stop the curvature progression, restore trunk balance (Goldberg 2002; Asher 2006) and prevent the long-term consequences of the deformity.

\section{Description of the intervention}

Interventions for the prevention of AIS progression include scoliosis-specific exercises, bracing and surgery (Rowe 1997; Lenssink 2005; Negrini 2005; Weiss 2006a), and other interventions have been reported in the literature. The goals ofALL interventions are to correct the deformity and prevent further deterioration of the curve (i.e. prevent progression) and to restore trunk asymmetry and balance, while minimising morbidity and pain, allowing return to full function (Bridwell 1999; Lonstein 2006).

Treatment approaches adopted by various orthopaedic surgeons and physicians specialising in the field of scoliosis around the world are divided, indicating lack of clinical equipoise across different 
professions and countries. In general, these approaches can be split grossly into two opposing groups. The first group consists of those who regard scoliosis-specific exercises as inefficient; members of the second group use these exercises and advocate their efficacy (Negrini 2005; Weiss 2006b). Similarly, bracing has been abandoned by some (Dolan 2007), but others support its use on the basis of existing weak evidence about efficacy (Negrini 2007); fusion is generally considered to be necessary when AIS exceeds a certain degree (approximately 45 to 50 degrees), when previous treatments have failed or when AIS causes symptoms, but indications vary widely according to the preference of the treating physician/surgeon (Dolan 2007).

These two conflicting approaches to conservative management seem to prevail in different regions of the world. In the United States, the United Kingdom and Australia, the wait-and-see strategy prevails, but in various parts of continental Europe and Eastern and Southern Europe, conservative treatment (scoliosis-specific exercises and bracing) is considered beneficial for the patient and is used routinely by a large majority of scoliosis physicians and surgeons (Lenssink 2005; Negrini 2005; Weiss 2006a). A possible reason for the negative beliefs toward scoliosis-specific exercises within the clinical community in the United Kingdom, in the United States and in some other countries is lack of knowledge within the physical therapy community and among associated clinical specialists. These pathological condition-specific exercises are not taught at an undergraduate or postgraduate level within the physiotherapy curriculum in both countries, and most clinicians (both physiotherapists and surgeons) in the United Kingdom normally do not appreciate the difference between scoliosisspecific exercises and general physiotherapy. Scoliosis-specific exercises consist of individually adapted exercises that are taught to patients in a centre that is totally dedicated to scoliosis treatment. Patients learn an exercise protocol that is personalized according to their own medical and physiotherapeutic evaluation. On the other hand, usual generalised physiotherapy is more generic, consisting of low-impact stretching and strengthening activities like yoga, pilates or tai chi (taiji), but it can include many different exercise protocols. Whilst scoliosis-specific exercises are usually used for treating mild curves of less than 25 to 30 degrees, they are also used frequently with braces to measure curves over this threshold. No side-effects of exercise are known, except for muscle soreness that can be felt if the intensity of exercise is too great (Weiss 2006a).

Bracing is defined as the application of external supports to the trunk; these are usually rigid and are applied with the aim of achieving maximum correction of the pathological curve (Rigo 2006a). Treatment commences when the curve is diagnosed as progressive, or when it exceeds a threshold of 30 degrees Cobb angle (Negrini 2005; Lonstein 2006; Weiss 2006a). Braces generally need to be worn for a considerable period of time per day (at least 20 hours), the treatment extending over several years until the end of bone growth, which usually occurs at 16 years of age for girls and 18 years of age for boys (Katz 2001). This causes a significant negative impact on the lives of children and adolescents (Fallstrom 1986; Noonan 1997; Climent 1999). Other conservative management strategies can be found in the literature: shoe insoles, electrotherapy and chiropractic treatment have all been reported. However, to date, there is a dearth of evidence for the effectiveness of these forms of therapy.

With regard to surgical interventions, a large multitude and variety are described in the literature (Maruyama 2008). These include different surgical approaches (anterior, posterior or combined) and many types of metal implants. The sophistication of spinal implants has grown rapidly in the past 10 to 15 years. Modern surgical techniques follow principles of segmental spinal instrumentation (this means that each vertebra of the spine is attached to a metal rod, wires or screws), and both anterior and posterior implants (surgical rods, wires or screws) are now available. Segmental instrumentation (with hooks or screws) can control sagittal and frontal plane correction in both lumbar and thoracic curves. In contrast to Harrington instrumentation, introduced in the $1960 \mathrm{~s}$ (Harrington 1962), segmental instrumentation allows early mobilisation of patients, thereby eliminating the need for postoperative casts and braces which were used in the past (Bradford 1987). This type of surgery also reduces the risk of potential neurological complications due to distraction forces (these are forces applied to a body part to separate bony fragments or joint surfaces) that were applied with the Harrington instrumentation (Harrington 1962). Countless studies have been published in the literature comparing different approaches to the spine (anterior, posterior or combined) and using various types of implants. A single threaded rod inserted through an anterior approach (from the front of the spine) was initially developed by Zielke, but this technique had a reported incidence of rod breakage as high as 31\% (Betz 1999). Further development of instrumentation resulted in the use of a double rod technique, such as Kaneda or Cotrel-Dubousset-Hopf, which prevented rod breakage but had the disadvantage of increasing the construct rigidity and favouring screw breakouts (Betz 2000). A further advantage was represented by a lower reoperation rate in double rod fixation (0\%) reported by Muschik 2006 as compared with single rod fixation (10\%; Betz 1999). The anterior approach is desirable because it can reliably correct curvature yet save the vertebral levels instrumented in lumbar or thoracolumbar curves (Arlet 2000; Kaneda 1996). However, if appropriate consideration is not given to planning and fusing the correct segments (i.e., to neutral and stable vertebrae), this can lead to curve progression and disc degeneration postoperatively (Bridwell 1994). Unfortunately, with the anterior approach to surgery, there is risk of potential trauma to the diaphragm and major abdominal organs. This type of surgery can also affect pulmonary function. If a patient has multiple curves, posterior fusion can achieve good correction and obviate the risks of anterior surgery (Bridwell 1999). The anterior approach also predisposes to a negative effect on pulmonary function for up to five years postoperatively (Kim 2005); therefore, some surgeons prefer a video-assisted thoracoscopy fol- 
lowed by posterior instrumentation, which allows them adequate spinal access but reduces the adverse effects on pulmonary function (Newton 2003).

Luhmann and Lenke (Luhman 2005) suggested that instrumentation through a posterior approach (approaching surgery from the back of the spine) was as efficient as a combined anterior and posterior approach, but the former (posterior approach) eliminated the negative effect on pulmonary function. In other words, approaching the surgical procedure from the back rather than from the front reduces the risks associated with deflating the lungs during the operative procedure (Di Silvestre 2008). A significant variety of implants and approaches to surgical treatment of the spine are available, but double rod posterior instrumentation seems to have become the preferred surgical intervention in cases where progression of scoliosis cannot be stopped by conservative treatment. All types of spinal fusion surgery are associated with significant risk both in the short term and in the long term. The short-term risk for spinal fusion surgery is estimated to be approximately $5 \%$, while long-term risks over a lifetime are estimated to exceed $50 \%$ (Weiss 2008a), with reoperation rates ranging from $6 \%$ to $20 \%$ (Asher 2006). However, reoperation rates may be very high (up to $50 \%$ ) with the use of more recent instrumentation such as CotrelDebusset instrumentation (Mueller 2012).

\section{How the intervention might work}

Scoliosis-specific exercises can be used in three main clinical scenarios: (1) the sole use of exercise as the primary treatment of AIS for mild curves, (2) in conjunction with braces for moderate curves and (3) during adulthood if the scoliosis curves exceed certain thresholds (Romano 2012). In the treatment of mild scoliosis, scoliosis-specific exercises can be used on curves greater than 10 to 15 degrees but less than 25 or 30 degrees Cobb. These intense

three-dimensional spine- and rib cage- specific exercises are used to try to limit the progression of the curve and thereby avoid the use of a brace. This critical Cobb angle is generally regarded as the threshold for brace prescription (Lonstein 2006; Weiss 2006c). In mild scoliosis cases for which exercise is prescribed, exercise is used predominantly according to the recommendations made by the Study Group on Scoliosis and Orthopaedic and Rehabilitative Treatment (SOSORT Guidelines 2012). The key objectives of physical exercise in mild cases of AIS include stabilisation of the spine combined with three-dimensional auto correction of the pelvis, rib cage and shoulders in combination with isometric muscle contractions (Romano 2012; Weiss 2006c).

Whilst scoliosis-specific exercises use internal corrective forces (i.e. muscles), braces use external corrective forces to correct the trunk; this is usually achieved with the use of rigid supports. However, some braces (called soft braces) are made of material similar to elastic bands and comparable with materials used in physical therapy treatments (Coillard 2003; Rigo 2006a). The mechanical forces of the brace are used to straighten the spine and derotate the pelvis and shoulders to bring the whole body into normal alignment. Negrini 2010 states that the external and proprioceptive inputs due to bracing change the unnatural loading on the spine and rib cage, decrease asymmetrical movements and improve neuromuscular control; this facilitates proper spinal growth, neuromotor reorganization and changes in motor behaviours (Coillard 2002; Lupparelli 2002; Castro 2003; Odermatt 2003; Weiss 2004; Negrini 2006c; Stokes 2006; Grivas 2008; Smania 2008). Unfortunately, most braces have the disadvantage of being not very comfortable to wear, especially for long periods. Further, if bracing is NOT combined with scoliosis-specific exercises, weakening of the back muscles may occur.

With regard to surgical treatment of the scoliotic spine, the two main approaches discussed previously (anterior and posterior) aim to correct the spinal curvature (reduction of the Cobb angle) and fuse the spine with the help of bone grafts that allow the spine to heal to a solid and stable bone fusion mass (spinal fusion), supported by the instrumentation (Haher 2003). Posterior spinal fusion with instrumentation and bone grafting is performed through the patient's back while the patient lies on his or her stomach. During this type of surgery, the surgeon attaches a metal rod to each side of the patient's spine (this can vary depending on the type of instrumentation and procedure used) by using hooks or screws attached to the vertebral bodies (Cailliet 1975). The surgeon then fuses the spine with a bone graft (this is a piece of bone that is usually taken from the patient's hip). The bone grows in between the vertebrae and holds them together, causing the parts of the spine that are fused to become like a rod. The metal rods attached to the spine ensure that the backbone remains straight while spinal fusion takes place (Cailliet 1975). The operation usually takes several hours. With recent advances in technology, patients can be discharged from the hospital within a week and do not require postoperative bracing. Many patients are able to return to school or work within 2 to 4 weeks after the surgery and resume all preoperative activities within 4 to 6 months. With the anterior approach, surgery is conducted through the chest wall instead of through the patient's back. The patient lies on his or her side during surgery. The surgeon places the incision on the patient's side, deflates the lung and removes a rib to reach the spine.There is worldwide general agreement that patients with curves in excess of 45 to 50 degrees are candidates for surgery. The selection of both instrumentation and operative procedure is dependent on curve location, magnitude and flexibility, as well as on sagittal alignment (Akbarnia 1988).

\section{Why it is important to do this review}

A scoping literature search identified only two systematic reviews on this topic. However, full methodological appraisals of the quality of included studies within these reviews was very limited (Hawes 2006a; Weiss 2008b). A systematic review conducted with 
state of the art, high-quality Cochrane methodology is urgently needed.

\section{O B JECT IVES}

The objective of this review is to evaluate and compare changes in trunk balance, progression of scoliosis, cosmetic issues, quality of life, disability, psychological issues and back pain, as well as adverse effects, with both surgical and non-surgical interventions provided in the short term (a few months) and in the long term (over 20 years).

\section{METHODS}

\section{Criteria for considering studies for this review}

\section{Types of studies}

The primary analysis will combine the results of randomised control trials (RCTs) and quasi-randomised control trials (QRCTs). We will also include prospective non-randomised studies (NRSs) with a control group because it is anticipated that very few RCTs will be found. We will include primary studies that compare surgical interventions with non-surgical interventions or no interventions (i.e. observation). We will exclude studies comparing nonsurgical methods alone (e.g. bracing vs scoliosis-specific exercises) as two other Cochrane reviews cover these questions Negrini 2010, Romano 2012).

\section{Types of participants}

The participants will be patients with AIS who were diagnosed and managed between 10 and 18 years of age, with a Cobb angle greater than 45 degrees (Scoliosis Research Society Guidance; accessed April). Studies on participants with early-onset scoliosis (infant or juvenile) or scoliosis secondary to other conditions will be excluded.

\section{Types of interventions}

The review will include all types of instrumented surgical interventions with fusion aimed to provide curve correction and spine stabilisation. Studies describing non-instrumented spinal correction and fusion will be excluded because it has been shown that they do not provide any better outcome than is seen with untreated scoliosis (Bradford 1987).

We aim to compare instrumented surgical interventions with different types of non-surgical treatments, such as scoliosis-specific exercises, bracing, physiotherapy, chiropractic treatment, electrical stimulation and other non-surgical interventions, as well as no treatment controls (i.e. observation).

\section{Types of outcome measures}

All outcomes (primary and secondary) will be measured in the immediate term (perioperative to six weeks postoperative), the short term (results at the end of bone growth), within two years, and over the long term (results in adulthood and in old age).

\section{Primary outcomes}

- Change in trunk balance, measured in centimetres:

- Frontal (coronal) balance (refers to the plane that divides the body into front and back halves);

- Lateral trunk shift; and

- Apical vertebral translation.

- Progression of scoliosis, measured by:

- Cobb angle in degrees (absolute values);

- Angle of trunk rotation (ATR) in degrees (absolute values); and

- Number of participants who have progressed by more than 5 degrees Cobb ( 5 degrees Cobb is the standard clinical measure reported within various research papers and commonly used in clinical practice).

\section{- Cosmetic issues, as measured by:}

- Validated scales or questionnaires: Walter Reed Visual Assessment Scale (WRVAS) (Pineda 2006), Spinal Appearance Questionnaire (SAQ) (Sanders 2007), Trunk Appearance Perception Scale (TAPS) (Bago 2010); and

- Topographic measurements: the integrated shape imaging system (ISIS) or ISIS2 (Berryman 2008), Quantec (Oxborrow 2000), Formetric (Knott 2010), measured in angles and millimetres.

\section{- Quality of life and disability:}

- Generic questionnaires: Short Form-36 (SF-36); and

- Scoliosis-specific questionnaires: SRS-22 (Asher 2003), Bad Sobernheim Stress Questionnaire (Weiss 2006c), Brace Questionnaire (Vasiliadis 2006).

\section{- Psychological issues:}

- Specific psychological questionnaires evaluating psychological concepts such as self-esteem, self-image etc., using specific questionnaires andsubscales of SRS-22, BrQ, SF-36.

\section{- Back pain and disability:}

- Validated scales measuring pain intensity and pain duration, such as the visual analogue scale, McGill Pain 
Questionnaire and other validated specific questionnaires, as well as use of medication.

\section{Secondary outcomes}

Secondary outcomes will include any adverse effects reported in the included studies. These may include blood loss, pseudarthrosis (a false joint where the bone has not healed adequately), deep wound infection, neurological complications, delayed Infections, pedicle screw misplacement, delayed paraparesis (weakness or partial paralysis in the lower limbs), loss of normal spinal function and decompensation (spinal imbalance) and increased spinal deformity, as well as death. If adverse effects are reported that are not listed here, we will still report them in our review.

\section{Search methods for identification of studies}

\section{Electronic searches}

We will search the following electronic databases and propose to search the literature since 1980 . We are not searching for papers before this date because a number of papers reporting the research on older instrumentation might not be relevant. Although clear advances in materials and design of spinal instrumentation have been made since 1980, the surgical approach and training might still be the same even though materials have changed.

- CENTRAL (The Cochrane Library, current issue).

- MEDLINE (1980 to present).

- EMBASE (1980 to present).

- CINAHL (1980 to present).

- PsycINFO (1980 to present).

- PEDro (1980 to present).

The search strategy combines the study design filter for observational studies adapted from the Scottish Intercollegiate Guidelines Network with the usual Cochrane RCT filter, so that all study designs will be captured by the search. The study design terms are combined with blocks of search terms for the disorder and the interventions. The strategy includes subject headings (e.g. $\mathrm{MeSH}$ ) and is adapted for the other databases (see Appendix 1 and Appendix 2).

\section{Searching other resources}

The following strategies will also be used.

- Screening the reference lists of all relevant papers.

- Searching the main electronic sources of ongoing trials (Cochrane Back Review Group Trials Register, National Research Register, meta-Register of Controlled Trials; Clinical Trials, World Health Organisation (WHO) International Clinical Trials Controlled Registry Platform).
- Searching the grey literature, including conference proceedings and $\mathrm{PhD}$ theses completed since 1980. For the latter, we will search the database, 'Dissertation Abstracts,' which lists American dissertations. This database also includes citations for dissertations from 50 British universities. To identify any further relevant British theses, we will search the Electronic Theses Online Service database (EThOS) provided by the British Library, which is an 'open access single point digital repository of UK research theses'.

- Contacting investigators and authors in this field for information on unpublished or incomplete trials.

All searches will include non-English language literature.

\section{Data collection and analysis}

\section{Selection of studies}

A data selection form will first be developed on the basis of inclusion criteria and will then be piloted and tested for both intraobserver and interobserver reliability by two review authors, who will then independently screen the search results by reading titles and abstracts. Potentially relevant studies will be obtained in full text and once again they will be independently assessed for inclusion by two review authors, who will resolve disagreement through discussion. A third review author will be contacted if disagreements persist. If a review author is also the author of a paper, another review author who has not authored any of the papers will undertake the selection.

\section{Data extraction and management}

A standardized data extraction form will be prepared on the basis of all inclusion criteria. This will first be piloted and tested for both intraobserver and interobserver reliability by two review authors, who then will independently use the form to extract raw data from the included papers. Raw data extraction will include study design (RCT, QRCT, prospective controlled cohort study), study characteristics (country, recruitment modality, study funding, risk of bias), participant characteristics (number of participants, age, sex, severity of scoliosis at baseline) and description of experimental and comparison interventions, cointerventions, adverse effects, duration of follow-up, outcomes assessed and results, as well as any adverse effects. If a review author is also the author of a paper, another review author will undertake the data extraction process. Any disagreement will be discussed and a third review author consulted if disagreements persist. 


\section{Assessment of risk of bias in included studies}

The risk of bias for both randomised studies and NRSs will be assessed using the criteria recommended by the Cochrane Back Review Group (Furlan 2009; Higgins 2011), together with items from the Downs and Black (Downs 1998) checklist, as outlined in Appendix 3. These criteria fall into five bias categories: selection bias, performance bias, attrition bias, detection bias and selective outcome reporting. The 'assessment of risk of bias' form will be piloted and tested for intraobserver and interobserver reliability. Two review authors will independently assess the internal validity of the included studies. Any disagreement between the review authors will be resolved by discussion; a third independent review author will be consulted if disagreements persist. Risk of bias assessment will be blinded to trial authors, institution and journal. The risk of bias criteria will be scored as high, low or unclear and will be reported in the 'risk of bias' table. The overall extent of risk of bias within each bias category (e.g. performance bias) will then be rated as "Bias" or "No bias".

Whilst it is difficult to provide an exhaustive list of all possible confounding variables at the start of the review, the review authors have experience in this field and are aware of most of the potential confounding variables that may occur when different treatment groups are compared. These may include, for instance, demographic variables such as age, Risser sign (bone maturity), curve location and curve magnitude.

When it comes to grading the quality of the evidence, evidence from studies judged "no bias" for all five categories will not be downgraded. Evidence will be downgraded (-1 point) when 3 or fewer categories for each study are judged to have bias. Evidence will be downgraded by -2 points when four or more categories for each study are judged to have bias. See the Data synthesis section that follows for additional details on quality assessment for each outcome.

\section{Measures of treatment effect}

Dichotomous outcomes will be analysed by calculating the risk ratio (RR) for each trial, with uncertainty in each result expressed by $95 \%$ confidence intervals (CIs). Continuous outcomes will be analysed by calculating the weighted mean difference (WMD) or the standardised mean difference (SMD) with 95\% CIs.

\section{Unit of analysis issues}

In cases where three or more interventions are evaluated in a single study, we will include each pairwise comparison separately.

\section{Dealing with missing data}

For recent papers (within 5 years), we will endeavour to collect missing data by contacting the authors. When data are insufficient to be entered into the meta-analysis (even after contacting the authors), we will report the results qualitatively in the 'table of characteristics of Included studies' and in the 'summary of findings tables'.

\section{Assessment of heterogeneity}

Outcome measures from individual trials will be combined through meta-analysis where possible (comparability of intervention and outcomes across trials) using a random-effects model. The $\mathrm{I}^{2}$ statistic and the $\mathrm{Chi}^{2}$ test $(\mathrm{P}<0.1)$ will be used to indicate whether significant statistical heterogeneity is present. If a metaanalysis is not possible, the results from clinically comparable trials will be described qualitatively in the text.

\section{Assessment of reporting biases}

To determine whether publication bias is present, we will construct funnel plots when at least 10 studies are available for the metaanalysis (Sutton 2000).

\section{Data synthesis}

Dichotomous outcomes will be analysed by calculating the RR. Continuous outcomes will be analysed by calculating the mean difference (MD) when the same instrument is used to measure outcomes or the SMD when different instruments are used to measure outcomes. Uncertainty will be expressed with 95\% CIs. Outcome measures from the individual trials will be combined through meta-analysis where possible (clinical comparability of population, intervention/s and outcomes between trials) using a random-effects model. A P value of the $\mathrm{Chi}^{2}$ test less than 0.1 will indicate significant statistical heterogeneity.

If meta-analysis is not possible, the results from clinically comparable trials will be described qualitatively in the text. Regardless of whether sufficient data are available for the use of quantitative analyses to summarise the data, we will assess the overall quality of the evidence for each outcome. To accomplish this, we will use the GRADE approach, as recommended in the Cochrane Handbook for Systematic Reviews of Interventions (Higgins 2011) and adapted in the updated Cochrane Back Review Group method guidelines (Furlan 2009). Factors that may decrease the quality of the evidence include study design and risk of bias, inconsistency of results, indirectness (not generalisable), imprecision (sparse data) and other factors (e.g. reporting bias).

The quality of the evidence for a specific outcome will be reduced by a level, according to the performance of the studies against these five factors.

High-quality evidence: Consistent findings have been noted among at least $75 \%$ of RCTs with low risk of bias; consistent, direct and precise data and no known or suspected publication biases. Further research is unlikely to change the estimate or our confidence in the results. 
Moderate-quality evidence: One of the domains is not met. Further research is likely to have an important impact on our confidence in the estimate of effect and may change the estimate.

Low-quality evidence: Two of the domains are not met. Further research is very likely to have an important impact on our confidence in the estimate of effect and is likely to change the estimate. Very low-quality evidence: Three of the domains are not met. We are very uncertain about the results.

No evidence: No studies were identified that addressed this outcome.

\section{Subgroup analysis and investigation of heterogeneity}

If significant statistical heterogeneity is noted, a subgroup analysis will be performed to consider the effects of the following variables: age, bone age, Cobb degrees, type of surgery and types of braces and exercise.

\section{Sensitivity analysis}

To incorporate the risk of bias assessment into the review process, we will start by stratifying the intervention effects estimates by risk. If differences in results are seen among studies at different risks of bias, we will go on to perform sensitivity analyses, excluding studies with high risk of bias from the analysis. Alternatively, we will present the results for RCTs and QRCTs separately from those of longitudinal studies.

\section{Clinical relevance of results}

The review authors will also assess each trial for its clinical relevance by using the five questions outlined by Shekelle 1994 and recommended by the Cochrane Back Review Group (Furlan 2009; Van Tulder 2003) (see Appendix 4). All important outcomes for each comparison will be discussed. The main conclusion will be clinical because our main aim is to give clinicians, researchers, patients and service users state-of-the-art information provided by relevant studies on this issue.

\section{ACKNOWLEDGEMENTS}

We would like to thank Rachel Couban, Cochrane Back Review Group Trials Search Co-ordinator for her help with development of the search strategies. We would also like to thank Dr Teresa Marin for her help with our queries in completing this protocol.

\section{R E F E R E N C E S}

\section{Additional references}

\section{Akbarnia 1988}

Akbarnia B. Selection of methodology in surgical treatment of adolescent idiopathic scoliosis. The Orthopedic Clinics of North America 1988;19(2):319-29.

\section{Arlet 2000}

Arlet V. Anterior thoracoscopic spine release in deformity surgery: a meta-analysis and review. European Spine Journal 2000;9(Suppl 1):S17-23.

\section{Asher 2003}

Asher M, Min Lai S, Burton D, Manna B. The reliability and concurrent validity of the Scoliosis Research Society- 22 patient questionnaire for idiopathic scoliosis. Spine 2003; 28(1):63-9.

\section{Asher 2006}

Asher M, Burton D. Adolescent idiopathic scoliosis: natural history and long term treatment effects. Scoliosis 2006;1:2. [DOI: $10.1186 / 1748-7161-1-2]$

Bago 2010

Bago J, Sanchez-Raya J, Sanchez Perez-Grueso F, Climent J. The Trunk Appearance Perception Scale (TAPS): a new tool to evaluate subjective impression of trunk deformity in patients with idiopathic scoliosis. Scoliosis 2010;5:6.

\section{Berryman 2008}

Berryman F, Pynsent P, Fairbank J, Disney S. A new system for measuring three-dimensional back shape in scoliosis. European Spine Journal 2008;7:663-72.

Betz 1999

Betz R, Harms J, Clements D III, Lenke L, Lowe T, Shufflebarger $\mathrm{H}$, et al.Comparison of anterior and posterior instrumentation for correction of adolescent thoracic idiopathic scoliosis. Spine 1999;24:225-39.

Betz 2000

Betz R, Lenke L, Lowe G. Proximal screw pull-out during anterior instrumentation for thoracic scoliosis: preventive techniques. 35th Annual Meeting Scoliosis Research Society. Cairns, 2000:156.

Bradford 1987

Bradford D, Lonstein J, Moe J, Ogilvie J, Winter R. Moe's Textbook of Scoliosis and Other Spinal Deformities. Philadelphia: Saunders, 1987.

\section{Bridwell 1994}

Bridwell K. Surgical treatment of adolescent idiopathic scoliosis: the basics and the controversies (Spine update). Spine 1994;19(9):1095-100.

Bridwell 1999

Bridwell K. Surgical treatment of idiopathic adolescent scoliosis. Spine (Focus Issue) 1999;24(24):2607-16. 


\section{Bunnell 1986}

Bunnell W. The natural history of idiopathic scoliosis before skeletal maturity. Spine 1986;11(8):773-6.

\section{Burwell 2000}

Burwell R, Dangerfield P. Adolescent idiopathic scoliosis: hypothesis of causation. State of the art reviews. Spine 2000;14(2):319-32.

Cailliet 1975

Cailliet R. Scoliosis: Diagnosis and Management. Philadelphia: FA Davis, 1975.

\section{Castro 2003}

Castro F Jr. Adolescent idiopathic scoliosis, bracing and the Hueter-Volkmann principle. Spine 2003;3(3):180-5.

\section{Clarisse 1974}

Clarisse P. Prognostic evolution of minor scoliosis curves of 10 degree to 29 degrees during the growth spurt [Prognostic evolutif des scolioses idiopathiques mineures de 10 degrees to 29 degrees en periode de croissance]. University Claude Bernard, Doctoral Thesis, Lyon 1974.

\section{Climent 1999}

Climent J, Sanchez J. Impact of the type of brace on the quality of life of adolescents with spine deformities. Spine 1999;24(18):1903-8.

\section{Coillard 2002}

Coillard C, Leroux M, Badeaux J, Rivard C. SPINECOR: a new therapeutic approach for idiopathic scoliosis. Studies in Health Technology and Informatics 2002;88:215-7.

\section{Coillard 2003}

Coillard C, Leroux M, Zabjek K, Rivard C. SpineCor- A nonrigid brace for the treatment of idiopathic scoliosis: post-treatment results. European Spine Journal 2003;12(2): $141-8$.

\section{Di Silvestre 2008}

Di Silvestre M, Bakaloudis G, Lolli F, Vommaro F, Martikos

$\mathrm{K}$, Parisini $\mathrm{P}$, et al.Posterior fusion only for thoracic adolescent idiopathic scoliosis of more than 80 degrees: pedicle screws versus hybrid instrumentation. European Spine Journal 2008;17:1336-49.

\section{Dickson 1984}

Dickson R, Lawton J, Archer I, Butt W. The pathogenesis of idiopathic scoliosis: biplanar spinal asymmetry. Journal of Bone and Joint Surgery 1984;66(1):8-15.

\section{Dolan 2007}

Dolan L, Weinstein S. Surgical rates after observation and bracing for adolescent idiopathic scoliosis: an evidencebased review. Spine 2007;32(19):S91-100.

\section{Downs 1998}

Downs $S$, Black N. The feasibility of creating a checklist for the assessment of the methodological quality both of randomised and non-randomised studies of health care interventions. Journal of Epidemiol Community Health 1998;52:377-84.

\section{Fallstrom 1986}

Fallstrom K, Cochran T, Nachemson A. Long-term effects on personality development in patients with adolescent idiopathic scoliosis: influence of type of treatment. Spine 1986;11(7):756-8.

\section{Freidel 2002}

Freidel K, Petermann F, Reichel D, Steiner A, Warschburger P, Weiss Hans R. Quality of life in women with idiopathic scoliosis. Spine 2002;27(4):E87-91.

Furlan 2009

Furlan A, Pennick V, Bombardier C, Van Tulder M, Editorial BCBRG. Updated method guidelines for systematic reviews in the Cochrane Back Review Group. Spine 2009;34(18):1929-41.

\section{Fustier 1980}

Fustier J. Spontaneous evolution of idiopathic scoliosis under 45 degrees during growth: retrospective study from the Lyon centre of rehabilitation [Evolution radiologique spontanee des scolioses idiopathiques de moins de 45 degrees en periode de croissance: etude graphique retrospective de cente dossier due Centre d'adaptation fanctionnelle de massues]. Thesis, University Claude Bernard, Lyon, 1980.

\section{Goldberg 2002}

Goldberg C, Moore D, Fogerty E, Dowling F. The natural history of early onset scoliosis. Studies in Health Technology and Informatics 2002;91:68-70.

\section{Grivas 2008}

Grivas T, Vasiliadis E, Olga D, Triantafyllopoulos S, Triantafyllopoulos G. What a school screening program could contribute in clinical research of idiopathic scoliosis aetiology. Disability and Rehabilitation 2008;30(10): 752-62.

\section{Haher 2003}

Haher T, Merola A. Atlas of Spine Surgical Techniques.. Atlas of Spine Surgical Techniques. New York, NY: Thieme, 2003.

\section{Harrington 1962}

Harrington P. Treatment of scoliosis: correction and internal fixation by instrumentation. Journal of Bone Joint Surgery 1962;44A:591-610.

\section{Hawes 2006a}

Hawes M. Impact of spine surgery on signs and symptoms of spinal deformity. Pediatric Rehabilitation 2006;9(4): 318-39.

\section{Hawes 2006b}

Hawes M, O'Brien J. The transformation of spinal curvature into spinal deformity: pathological processes and implications for treatment. Scoliosis 2006;1(3):1-9.

Higgins 2011

Higgins JPT, Green S (editors). Cochrane Handbook for Systematic Reviews of Interventions Version 5.1.0 [updated March 2011]. The Cochrane Collaboration, 2011. www.cochrane-handbook.org. Chichester: John Wiley and Sons.

Kanayama 1996

Kanayama M, Tadano S, Kaneda K, Ukai T, Abumi K. A. mathematical expression of three dimensional configuration of the scoliotic spine. ASME Journal of Biomechanical Engineering 1996;2(118):247-252. 


\section{Kaneda 1996}

Kaneda K, Kiyoshi M, Shono Y, Yasuhiro M, Satoh S, Shigenobu M, et al.New anterior instrumentation for the management of thoracolumbar and lumbar scoliosis: application of the Kaneda two-rod system. Spine 1996;21 (10):1250-61.

\section{Katz 2001}

Katz D, Durrani A. Factors that influence outcome in bracing large curves in patients with adolescent idiopathic scoliosis. Spine 2001;26(21):2354-61.

\section{Kim 2005}

Kim Y, Lenke L, Bridwell K, Kim K, Steger-May K. Pulmonary function in adolescent idiopathic scoliosis relative to the surgical procedure. Journal of Bone and Joint Surgery 2005;87:1534-41.

\section{Knott 2010}

Knott P, Mardjetko S, Rollet M, Baute S, Riemenschneider, Muncie L, et al.Evaluation of the reproducibility of the formetric 4D measurements for scoliosis. Scolisis 2010:(5 Suppl 1):O10 .

\section{Lenssink 2005}

Lenssinck M, Frijlink A, Berger Y, Bierman-Zeinstra S, Verkerk K, Verhagen A, et al.Effect of bracing and other conservative interventions in the treatment of idiopathic scoliosis in adolescents: a systematic review of clinical trials. Physical Therapy 2005;85(12):1329-39.

\section{Lonstein 1984}

Lonstein J, Carlson J. The prediction of curve progression in untreated idiopathic scoliosis during growth. Journal of Bone and Joint Surgery 1984;66(7):1061-71.

\section{Lonstein 2006}

Lonstein J. Scoliosis: surgical versus non-surgical treatment. Clinical Orthopaedics and Related Research 2006;443:28459.

\section{Luhman 2005}

Luhmann S, Lenke L, Kim Y, Bridwell K, Schootman M. Thoracic adolescent idiopathic scoliosis curves between 70 degrees and 100 degrees. Is anterior release necessary?. Spine 2005;30:2061-67.

\section{Lupparelli 2002}

Lupparelli S, Pola E, Pitta L, Mazza O, De Santis V, Aulisa $\mathrm{L}$, et al.Biomechanical factors affecting progression of structural scoliotic curves of the spine. Studies in Health Technology and Informatics 2002;91:81-5.

\section{Machida 1999}

Machida M. Cause of idiopathic scoliosis. Spine 1999;24 (24):2576-83.

\section{Maruyama 2008}

Maruyama T, Takeshita K. Surgical treatment of scoliosis: a review of techniques currently applied. Scoliosis 2008;3(6): $1-6$.

\section{Mehta 1972}

Mehta M. The rib-vertebra angle in the early diagnosis between resolving and progressive infantile scoliosis. Journal of Bone and Joint Surgery 1972;54(2):230-43.

\section{Mueller 2012}

Mueller F, Gluch H. Cotrel-Dubousset instrumentation for the correction of adolescent idiopathic scoliosis: long-term results with an unexpected high revision rate. Scoliosis 2012; $7(1): 13$.

\section{Muschik 2006}

Muschik M, Kimmich H, Demmel T. Comparison of anterior and posterior double-rod instrumentation for thoracic idiopathic scoliosis: results of 141 patients. European Spine Journal 2006;15:1128-38.

\section{Negrini 2005}

Negrini S, Aulisa L, Ferraro C, Fraschini P, Masiero S, Simonazzi $\mathrm{P}$, et al.Italian guidelines on rehabilitation treatment of adolescents with scoliosis or other spinal deformities. Europa Medicophysica 2005;41(2):183-201.

\section{Negrini 2006c}

Negrini S, Marchini G. Efficacy of the symmetric, patientoriented, rigid, three-dimensional, active (SPoRT) concept of bracing for scoliosis: a prospective study of the Sforzesco versus Lyon brace. Europa Medicophysica 2006;43(2): $171-81$.

\section{Negrini 2007}

Negrini S, Marchini G. Efficacy of the symmetric, patientoriented,rigid, three-dimensional, active (SPoRT) concept of bracing for scoliosis: a prospective study of the Sforzesco versus Lyon brace. Europa Medicophysica 2007;43(2): 171-81.

\section{Negrini 2010}

Negrini S, Minozzi S, Romano M, Bettany-Saltikov JA, Chokalingham N, Grivas T, Maruyama T . Braces for idiopathic scoliosis in adolescents. Cochrane Database of Systematic Reviews 2010, Issue Issue 1. [DOI: 10.1002/ 14651858.CD006850.pub2]

\section{Newton 2003}

Newton P, Marks M, Faro F, Betz R, Clements D, Haher T, et al.Use of video-assisted thoracoscopic surgery to reduce perioperative morbidity in scoliosis surgery. Spine 2003;28: S249-54.

\section{Noonan 1997}

Noonan K, Dolan L, Jacobson W, Weinstein S. Long-term psychosocial characteristics of patients treated for idiopathic scoliosis. Journal of Pediatric Orthopaedics 1997;17(6): 712-7.

\section{Odermatt 2003}

Odermatt D, Mathieu P, Beausejour M, Labelle H, Aubin C. Electromyography of scoliotic patients treated with a brace. Journal of Orthopaedic Resources 2003;21(5):931-6.

\section{Oxborrow 2000}

Oxborrow N. Methodology. Current topic: assessing the child with scoliosis: the role of surface topography. Archives of Disease in Childhood 2000;83:453-5 .

\section{Perdriolle 1985}

Perdriolle R, Vidal J. Thoracic idiopathic scoliosis: curve evolution and prognosis. Spine 1985;10(9):785-91. 


\section{Pineda 2006}

Pineda S, Bago J, Gilperez C, Climent J. Validity of the Walter Reed Visual Assessment Scale to measure subjective perception of spine deformity in patients with idiopathic scoliosis. Scoliosis 2006;1:18.

Reichel 2003

Reichel D, Schanz J. Developmental psychological aspects of scoliosis treatment. Pediatric Rehabilitation 2003;6(34): $221-5$.

\section{Rigo 2006a}

Rigo M, Negrini S, Weiss H, Grivas T, Maruyama T, Kotwicki T, et al.SOSORT consensus paper on brace action: TLSO biomechanics of correction (investigating the rationale for force vector selection). Scoliosis 2006;1:11.

Roaf 1980

Roaf R. Spinal Deformities, 2nd edition. Tunbridge Wells: Pitman Medical, 1980.

\section{Rogala 1978}

Rogala E, Drummond D, Gurr J. Scoliosis: incidence and natural history. A prospective epidemiological study. Journal of Bone and Joint Surgery 1978;60(2):173-6.

Romano 2012

Romano M, Minozzi S, Bettany-Saltikov J, Zaina F, Chockalingam N, Kotwicki T, et al.Exercises for adolescent idiopathic scoliosis. Cochrane Database of Systematic Reviews 2012, Issue 8. [DOI: 10.1002/ 14651858.CD007837.pub2]

Rowe 1997

Rowe D, Bernstein S, Riddick M, Adler F, Emans J, Gardner-Bonneau D, et al.A meta-analysis of the efficacy of non-operative treatments for idiopathic scoliosis. Journal of Bone and Joint Surgery, American 1997;79(5):664-74.

\section{Sanders 2007}

Sanders J, Harrast J, Kuklo T, Polly D, Bridwell K, Diab M, et al.The Spinal Appearance Questionnaire: results of reliability, validity, and responsiveness testing in patients with idiopathic scoliosis. Spine 2007;32(24):2719-22.

\section{Sevastik 1997}

Sevastik B, Xiong B, Sevastik J, Lindgren U, Willers U. Ribvertebral angle asymmetry in idiopathic, neuromuscular and experimentally induced scoliosis.. European Spine Journal 1997;6:84-8.

\section{Shekelle 1994}

Shekelle P, Andersson G, Bombardier C, Cherkin D, Deyo $\mathrm{R}$, Keller R, et al.A brief introduction to the critical reading of the clinical literature. Spine 1994;19(19):2028S-31S.

Smania 2008

Smania N, Picelli A, Romano M, Negrini S.

Neurophysiological basis of rehabilitation of adolescent idiopathic scoliosis. Disability and Rehabilitation 2008;30 (10):763-71.

SOSORT Guidelines 2012

Negrini S, Aulisa A, Aulisa L, Circo A, De Mauroy J, Durmala J, et al.SOSORT guidelines: orthopaedic and rehabilitation treatment of idiopathic scoliosis during growth. Scoliosis SOSORT Guidelines 2012; Vol. 7, issue $1: 3$.

Stokes 1996

Stokes I, Spence H, Aronsson D, Kilmer N. Mechanical modulation of vertebral body growth: implications for scoliosis progression. Spine 1996;21(10):1162-7.

Stokes 1997

Stokes I. Analysis of symmetry of vertebral body loading consequent of lateral spinal curvature. Spine 1997;22: 2495-503.

Stokes 2006

Stokes I, Burwell R, Dangerfield P. Biomechanical spinal growth modulation and progressive adolescent scoliosis- a test of the 'vicious cycle' pathogenetic hypothesis: summary of an electronic focus group debate of the IBSE. Scoliosis 2006;1:16.

Sutton 2000

Sutton A, Duval S, Tweedie R, Abrams K, Jones D. Empirical assessment of effect of publication bias on metaanalyses. British Medical Journal 2000;320:1574-7.

\section{Van Tulder 2003}

Van Tulder M, Furlan A, Bombardier C, Bouter L, Editorial Board Cochrane Back Review Group. Updated method guidelines for systemic reviews in the Cochrane Collaboration Back Review Group. Spine 2003;28(12): 1290-9.

Vasiliadis 2006

Vasiliadis E, Grivas T, Gkoltsiou K. Development and preliminary validation of Brace Questionnaire (BrQ): a new instrument for measuring quality of life of brace treated scoliotics. Scoliosis 2006;1(7):1-8.

\section{Weinstein 1983}

Weinstein S, Ponseti I. Curve progression in idiopathic scoliosis. Journal of Bone and Joint Surgery 1983;65(4): 447-55.

\section{Weiss 2008a}

Weiss H, Goodall D. Rate of complications in scoliosis surgery: a systematic review of the PubMed literature. Scoliosis 2008;3:1-18. [DOI: 10.1186/1748-7161-3-9]

\section{Weiss 2004}

Weiss H, Hawes M. Adolescent idiopathic scoliosis, bracing and the Hueter-Volkmann principle. Spine Journal 2004;4 (4):485-6.

\section{Weiss 2006a}

Weiss H, Negrini S, Hawes M, Rigo M, Kotwicki T, Grivas $\mathrm{T}$, et al.Physical exercises in the treatment of idiopathic scoliosis at risk of brace treatment. SOSORT Consensus Paper Scoliosis 2006;1:6.

\section{Weiss 2006}

Weiss H, Negrini S, Rigo M, Kotwicki T, Hawes M, Grivas $\mathrm{T}$, et al.Indications for conservative management of scoliosis (guidelines). Scoliosis 2006;1:5. 


\section{Weiss 2006c}

Weiss H, Reichel D, Schanz J, Zimmermann-Gudd S.

Deformity related stress in adolescents with AIS. Studies of Health and Technology Informatics 2006;123:347-51.

\section{Weiss 2008b}

Weiss H. Adolescent idiopathic scoliosis (AIS)- An indication for surgery? A systematic review of the literature. Disability and Rehabilitation 2008;30(10):799-807.

\section{Weiss 2012}

Weiss H. Physical therapy intervention studies on idiopathic scoliosis: review with the focus on inclusion criteria.

Scoliosis 2012;7(4):1-11. [DOI: 10.1186/1748-7161-7-4]

\section{White 1990}

White A, Panjabi M. Functional analysis and clinical applications. Chapter 7. In: Clinical Biomechanics of the Spine. 2nd Edition. Baltimore: Lippincott, 1990.

* Indicates the major publication for the study

\section{A P P E N DICES}

\section{Appendix I. Medline search strategy}

1. exp Spinal Diseases/

2. Scoliosis/

3. scoliosis

4. or $/ 1-3$

5. Orthopedics/

6. exp Surgical Procedures, Operative/

7. su.fs.

8. surg\$.tw.

9. operat\$.tw.

10. realign\$.tw.

11. spondylodesis.tw.

12. spine fusion.tw.

13. spinal fusion.tw.

14. spinal instrumentation.tw.

15. Bone Screws/

16. screw\$.tw.

17. hybrid.tw.

18. Bone Wires/

19. sublaminar.tw.

20. wire\$.tw.

21. hook\$.tw.

22. or $/ 5-21$

23. exp Rehabilitation/

24. rehabilit\$.tw.

25. rh.fs.

26. exp Physical Therapy Modalities/

27. Physical Therapy Speciality

28. physiotherapy.tw.

29. physical therapy.tw.

30. exp Exercisel

31. exercise\$.tw.

32. Exercise Movement Techniques/

33. exp Exercise Therapy/

34. exp Musculoskeletal Manipulations/

35. Immobilization/ 
36. Braces/

37. brace $\$ . m p$.

38. bracing.mp.

39. exp Othotic Devices/

40. Orthopedic Equipment/

41. limit 40 to $\mathrm{yr}=" 1902-1975 "$

42. (non-surg $\$$ or nonsurg $\$$ or non-operat $\$$ or nonoperat $\$$ or conserv $\$$ ).tw.

43. (immobilis $\$$ or immobiliz $\$$ or therap $\$$ or taping or tape $\$$ or electrotherapy $\$$ ).tw.

44. or $/ 23-43$

45. 4 and 22 and 44

46. limit 45 to adolescent $<13$ to 18 years $>$

47. Adolescent/

48. adolescen $\$$

49. 47 or 48

50. 45 and 49

51. 46 or 50

52. Comparative Study/

53. exp Evaluation Studies/

54. exp Follow-up Studies/

55. exp Prospective Studies/

56. $\exp$ Cross-over Studies/

57. exp Epidemiologic Studies/

58. exp Case-Control Studies/

59. exp Cohort Studies/

60. exp Cross-Sectional Studies/

61. (cohort adj (study or studies)).mp.

62. cohort analy\$.mp.

63. (follow up adj (study or studies)).mp.

64. (observational adj (study or studies)).mp.

65. longditundinal.mp.

66. retrospective.mp.

67. cross sectional.mp.

68. control\$.mp.

69. prospective\$.mp.

70. volunteer.mp.

71. or $/ 52-70$

72. randomized controlled trial.pt.

73. controlled clinical trial.pt.

74. randomized.ti,ab.

75. placebo.ti,ab.

76. drug therapy.fs.

77. randomly.ti,ab.

78. trial.ti,ab.

79. groups.ti,ab.

80. or/72-79

81. (Animals not (Humans and Animals)).sh.

82. 80 not 81

83. Animals/

84. Humans/

85. 83 not (83 and 84 )

86. 80 not 85

87. 71 not 85

88. 82 or 86 or 87

Surgical versus non-surgical interventions in patients with adolescent idiopathic scoliosis (Protocol)

Copyright $\odot 2013$ The Cochrane Collaboration. Published by John Wiley \& Sons, Ltd. 
89. 51 and 88

\section{Appendix 2. other search strategies}

CINAHL

S1 MH Clinical Trials+

S2 "randomi?ed controlled trial"”

S3 clinical W3 trial

S4 double-blind

S5 single-blind

S6 triple-blind

S7 TI groups or AB groups

S8 MH Placebo Effect

S9 MH Placebos

S10 placebo*

S11 MW Drug Therapy

S12 random*

S13 MH Random Sample

$\mathrm{S} 14 \mathrm{~S} 1$ or $\mathrm{S} 2$ or $\mathrm{S} 3$ or $\mathrm{S} 4$ or $\mathrm{S} 5$ or $\mathrm{S} 6$ or $\mathrm{S} 7$ or $\mathrm{S} 8$ or $\mathrm{S} 9$ or $\mathrm{S} 10$ or $\mathrm{S} 11$ or $\mathrm{S} 12$ or S13

S15 MH Study Design+

S16 latin square

S17 MH Comparative Studies

S18 MH Evaluation Research+

S19 MH Prospective Studies+

S20 MH Epidemiological Research+

S21 "cohort studies" or "cohort study"

S22 "cohort analys"”

S23 "follow-up stud" or "followup stud"”

S24 "observational studies" or "observational study"

S25 longitudinal

S26 retrospective

S27 control $^{*}$

S28 prospective*

S29 volunteer*

S30 S15 or S16 or S17 or S18 or S19 or S20 or S21 or S22 or S23 or S24 or S25

or $\mathrm{S} 26$ or $\mathrm{S} 27$ or $\mathrm{S} 28$ or $\mathrm{S} 29$

S31 MH Animals

S32 S14 not S31

S33 S30 not S31

S34 S32 or S33

S35 MH Spine+

S36 MH Spinal Diseases+

S37 MH Scoliosis+

S38 scoliosis

S39 S35 or S36 or S37 or S38

S40 MH Orthopedics

S41 MH Surgery, Operative+

S42 MW Surgery

S43 TI surg* or AB surg*

S44 TI operat* or AB operat*

S45 TI realign* or AB realign* 
S46 TI spondylodesis or AB spondylodesis

S47 TI "spine fusion" or AB "spine fusion"

S48 TI "spinal fusion" or AB "spinal fusion"

S49 TI "spinal instrumentation" or AB "spinal instrumentation"

S50 MH Orthopedic Fixation Device

S51 TI screw* or AB screw*

S52 TI hybrid or AB hybrid

S53 TI sublaminar or $\mathrm{AB}$ sublaminar

S54 TI wire* or AB wire*

S55 TI hook* or AB hook*

S56 S40 or S41 or S42 or S43 or S44 or S45 or S46 or S47 or S48 or S49 or S50

or $\mathrm{S} 51$ or $\mathrm{S} 52$ or $\mathrm{S} 53$ or $\mathrm{S} 54$ or $\mathrm{S} 55$

S57 MH Rehabilitation+

S58 MH Physical Therapy+

S59 MH Physical Therapists

S60 TI physiotherapy or $\mathrm{AB}$ physiotherapy

S61 TI "physical therapy" or AB "physical therapy"

S62 MH Exercise+

S63 TI exercise* or AB exercise*

S64 MH Therapeutic Exercise+

S65 MH Manipulation, Orthopedic

S66 MH Immobilization

S67 brace*

S68 bracing

S69 orthoses

S70 orthotic*

S71 MH "Orthopedic Equipment and Supplies+"

S72 TI (non-surg* or nonsurg* or non-operat* or nonoperat* ${ }^{*}$ or conserv*) or

$\mathrm{AB}$ (non-surg* or nonsurg* or non-operat* or nonoperat* or conserv*)

S73 TI (immobilis* or immobiliz* or therap* $^{*}$ or taping or tape* or electrotherap*) or

$\mathrm{AB}$ (immobilis* or immobiliz* or therap* or taping or tape* or electrotherap*)

S74 S57 or S58 or S59 or S60 or S61 or S62 or S63 or S64 or S65 or S66 or S67

or $\mathrm{S} 68$ or $\mathrm{S} 69$ or $\mathrm{S} 70$ or $\mathrm{S} 71$ or $\mathrm{S} 72$ or $\mathrm{S} 73$

S75 S39 and S56 and S74

S76 S39 and S56 and S74 Limiters - Age Groups: Adolescent: 13-18 years

S77 MH Adolescence+

S78 adolescen*

S79 S77 or S78

S80 S75 and S79

S81 S76 or S80 (without filter)

S82 S34 and S81 (applies filter)

S83 S81 not S82 (checks what has been lost)

EMBASE

1 exp spine/

2 exp spine disease/

3 exp scoliosis/

4 exp idiopathic scoliosis/

5 scoliosis.mp.

6 or/ $1-5$

7 orthopedics/

8 exp surgery/

9 su.fs.

$10 \operatorname{surg} \$ . t i, a b$.

Surgical versus non-surgical interventions in patients with adolescent idiopathic scoliosis (Protocol)

Copyright $\odot 2013$ The Cochrane Collaboration. Published by John Wiley \& Sons, Ltd. 
11 operat $\$ . t i, a b$.

12 realign $\$ . t i, a b$.

13 spondylodesis.ti,ab.

14 spine fusion.ti,ab.

15 spinal fusion.ti,ab.

16 spinal instrumentation.ti,ab.

17 bone screw/

18 screw\$.ti,ab.

19 hybrid.ti,ab.

20 Kirschner wire/

21 sublaminar.ti,ab.

22 wire \$.ti,ab.

23 hook\$.ti,ab.

24 or/ $7-23$

25 exp rehabilitation/

26 rehabilitat $\$ . t i, a b$.

27 rh.fs.

28 exp physiotherapy/

29 physiotherapist/

30 physiotherapy.ti,ab.

31 physical therapy.ti,ab.

32 exp exercise/

33 exercise $\$ . t i, a b$.

34 kinesiotherapy/

35 exp manipulative medicine/

36 immobilization/

37 brace/

38 brace $\$ . m p$.

39 bracing.mp.

40 exp orthotics/

41 exp orthopedic equipment/

42 (non-surg\$ or nonsurg\$ or non-operat $\$$ or nonoperat $\$$ or conserv\$).ti,ab.

43 (immobilis\$ or immobiliz\$ or therap\$ or taping or tape $\$$ or electrotherap\$).ti,ab.

44 or $/ 25-43$

456 and 24 and 44

46 limit 45 to adolescent $<13$ to 17 years $>$

47 adolescent/

48 adolescen $\$$.mp.

49 or/ $47-48$

$50 \quad 45$ and 49

5146 or 50

52 exp clinical study/

53 exp case control study/

54 family study/

55 longitudinal study/

56 retrospective study/

57 prospective study/

58 cohort analysis/

59 (cohort adj (study or studies)).mp.

60 (case control adj (study or studies)).mp.

61 (follow up adj (study or studies)).mp.

62 (observational adj (study or studies)).mp.

63 (epidemiologic\$adj (study or studies)).mp.

Surgical versus non-surgical interventions in patients with adolescent idiopathic scoliosis (Protocol)

Copyright $\odot 2013$ The Cochrane Collaboration. Published by John Wiley \& Sons, Ltd. 
64 (cross sectional adj (study or studies)).mp.

65 exp comparative study/

66 evaluation study.mp.

67 follow-up study.mp. or exp follow up/

68 crossover procedure/

69 prospectiv $\$ . m p$.

70 volunteer/

71 or $/ 52-70$

72 clinical article/

73 exp clinical study/

74 clinical trial/

75 controlled study/

76 randomized controlled trial/

77 major clinical study/

78 major clinical study/

79 double blind procedure/

80 multicenter study/

81 single blind procedure/

82 phase 3 clinical trial/

83 phase 4 clinical trial/

84 placebo/

85 or/72-84

86 allocat\$.mp.

87 assign \$.mp.

88 blind\$.mp.

89 (clinic\$ adj25 (study or trial)).mp.

90 compar\$.mp.

91 control\$.mp.

92 cross?over.mp.

93 factorial\$.mp.

94 follow?up.mp.

95 placebo\$.mp.

96 prospectiv\$.mp.

97 random\$.mp.

98 ((singl\$ or doubl\$ or trebl\$ or tripl\$) adj25 (blind\$ or mask\$)).mp.

99 trial.mp.

100 (versus or vs).mp.

101 or/86-100

10285 and 101

10371 or 102

104 human/

105 nonhuman/

106 exp animal/

107 animal experiment/

108 or/105-107

109104 not 108

110103 not 108

111 or/109-110

11251 and 111

PEDro

Abstract \& Title: scoliosis

AND

Method: clinical trial

Surgical versus non-surgical interventions in patients with adolescent idiopathic scoliosis (Protocol)

Copyright $\odot 2013$ The Cochrane Collaboration. Published by John Wiley \& Sons, Ltd. 
PsycInfo

S1 scoliosis

S2 surg* or operat* or realign* or spondylodesis or fusion or instrumentation or

screw* or hook* or hybrid or wire* or sublaminar

S3 rehabilit* or therap* ${ }^{*}$ or physiotherapy or exercise* or braces or bracing or

orthotic* or non-surg* $^{*}$ or nonsurg* or non-operat* ${ }^{*}$ or nonoperat* ${ }^{*}$ or conserv* ${ }^{*}$ or

immobilis* or immobiliz* or taping or tape* or electrotherapy

S4 S1 and S2 and S3

CBRG Trials Register in CLIB

\#1 (SR-BACK) in Trials

\#2 scoliosis

\#3 (\#1 AND \#2)

ClinicalTrialsdotgov

Search term: scoliosis

WHO ICTRP

Condition: scoliosis

\section{Appendix 3. Criteria for assessing risk of bias for internal validity for randomised and non- randomised studies (Downs and Black 1998; Furlan 2009)}

\section{Selection bias}

\section{Random sequence generation}

Risk of selection bias is low if the investigators describe a random component in the sequence generation process, such as referring to a random number table, using a computer random number generator, coin tossing, shuffling cards or envelopes, throwing dice, drawing lots, minimising (minimisation may be implemented without a random element, and this is considered to be equivalent to being random).

Risk of selection bias is high if the investigators describe a non-random component in the sequence generation process, such as sequence generated by odd or even date of birth, date (or day) of admission, hospital or clinic record number or allocation by judgement of the clinician, preference of the participant, results of a laboratory test or a series of tests or availability of the intervention.

If it is a non-randomised study, this will be rated as high bias.

\section{Allocation concealment}

Risk of selection bias is low if participants and investigators enrolling participants could not foresee assignment because one of the following, or an equivalent method, was used to conceal allocation: central allocation (including telephone, Web-based and pharmacycontrolled randomisation); sequentially numbered drug containers of identical appearance; or sequentially numbered, opaque, sealed envelopes.

Risk of bias is high if participants or investigators enrolling participants could possibly foresee assignments and thus introduce selection bias, such as allocation based on using an open random allocation schedule (e.g. a list of random numbers); assignment envelopes were used without appropriate safeguards (e.g. if envelopes were unsealed or non-opaque or were not sequentially numbered); alternation or rotation; date of birth; case record number; or other explicitly unconcealed procedures.

If it is a non-randomised study, this will be rated as high bias.

\section{Selection bias (population)*}

Risk of selection bias is low if participants in different intervention groups are recruited from the same population.

Selection bias (timing)*

Risk of selection bias is low if participants in different intervention groups are recruited over the same time. Surgical studies must be $<10$ years old for low risk of selection bias.

\section{Adjustment for confounding*}

Risk is low if no significant group differences were shown. Risk is high if the effect of the main confounders was not investigated or if no adjustment was made in the final analyses.

Surgical versus non-surgical interventions in patients with adolescent idiopathic scoliosis (Protocol) 


\section{Performance bias}

\section{Blinding of participants}

Risk of performance bias is low if blinding of participants was ensured and it was unlikely that the blinding could have been broken; or if no blinding or incomplete blinding was performed, but the review authors judge that the outcome is not likely to be influenced by lack of blinding.

\section{Blinding of personnel/ care providers}

Risk of performance bias is low if blinding of personnel was ensured and it was unlikely that the blinding could have been broken; or if no blinding or incomplete blinding was performed, but the review authors judge that the outcome is not likely to be influenced by lack of blinding.

Compliance (adherence)

Risk of bias is low if compliance with the interventions was acceptable on the basis of reported intensity/dosage, duration, number and frequency for both index and control intervention(s). For single-session interventions (e.g. surgery), this item is irrelevant.

\section{Cointerventions}

Risk of bias is low if no cointerventions were provided, or if they were similar between index and control groups.

\section{Attrition bias}

\section{Incomplete outcome data}

Risk of attrition bias is low if no outcome data were missing; reasons for missing outcome data were unlikely to be related to the true outcome (for survival data, censoring unlikely to be introducing bias); missing outcome data were balanced in numbers, with similar reasons for missing data across groups; for dichotomous outcome data, the proportion of missing outcomes compared with the observed event risk was not enough to have a clinically relevant impact on the intervention effect estimate; for continuous outcome data, the plausible effect size (difference in means or standardised difference in means) among missing outcomes was not enough to have a clinically relevant impact on observed effect size, or missing data were imputed using appropriate methods (if dropouts are very large, imputation using even "acceptable" methods may still suggest a high risk of bias). The percentage of withdrawals and dropouts should not exceed 20\% for short-term follow-up and 30\% for long-term follow-up and should not lead to substantial bias (these percentages are commonly used but arbitrary and are not supported by the literature).

Intention-to-treat analysis

Risk of bias is low if all randomly assigned participants were reported/analysed in the group to which they were allocated by randomisation.

\section{Measurement/detection}

\section{Blinding of outcome assessment}

Risk of detection bias is low if blinding of the outcome assessment was ensured and it was unlikely that the blinding could have been broken; or if no blinding or incomplete blinding was performed, but the review authors judge that the outcome is not likely to be influenced by lack of blinding, or:

- for participant-reported outcomes in which the participant was the outcome assessor (e.g. pain, disability): Risk of bias for outcome assessors is low if risk of bias for participant blinding is low;

- for outcome criteria that are clinical or therapeutic events that will be determined by the interaction between participants and care providers (e.g. cointerventions, length of hospitalisation, treatment failure), in which the care provider is the outcome assessor: Risk of bias for outcome assessors is low if risk of bias for care providers is low; and

- for outcome criteria that are assessed from data from medical forms: Risk of bias is low if the treatment or adverse effects of the treatment could not be noticed in the extracted data.

\section{Timing of outcome assessments}

Risk of bias is low if all important outcome assessments for all intervention groups were measured at the same time, or if analyses adjust for different lengths of follow-up.

\section{Selective reporting}

Data dredging

Risk of bias is low if all analyses were planned at the outset of the study.

Surgical versus non-surgical interventions in patients with adolescent idiopathic scoliosis (Protocol)

Copyright @ 2013 The Cochrane Collaboration. Published by John Wiley \& Sons, Ltd. 
Risk of bias is high if analyses were conducted retrospectively (e.g. retrospective unplanned subgroup analyses).

Outcome measures

Risk of reporting bias is low if the study protocol is available and all of the study's prespecified (primary and secondary) outcomes that are of interest in the review have been reported in the prespecified way, or if the study protocol is not available, but it is clear that the published reports include all expected outcomes, including those that were prespecified (convincing text of this nature may be uncommon).

Risk of reporting bias is high if not all of the study's prespecified primary outcomes have been reported; one or more primary outcomes are reported using measurements, analysis methods or subsets of the data (e.g. subscales) that were not prespecified; one or more reported primary outcomes were not prespecified (unless clear justification for their reporting is provided, such as an unexpected adverse effect); one or more outcomes of interest in the review are reported incompletely, so that they cannot be entered into a meta-analysis; the study report fails to include results for a key outcome that would be expected to have been reported for such a study.

*Items are relevant only to non-randomised studies.

\section{Appendix 4. Questions for assessing clinical relevance}

1. Are the participants described in detail so that you can decide whether they are comparable with those that you see in your practice?

Yes/No/Unsure

2. Are the interventions and treatment settings described well enough that you can provide the same for your patients?

Yes/No/Unsure

3. Were all clinically relevant outcomes measured and reported?

Yes/No/Unsure

4. Is the size of the effect clinically important?

Yes/No/Unsure

5. Are the likely treatment benefits worth the potential harms?

Yes/No/Unsure

\section{CONTRIBUTIONS OF AUTHORS}

Substantial contributions to conception and design: Josette Bettany-Saltikov, Hans-Rudolf Weiss, Razvan Taranu, Shreya Srinivas, Nachiappan Chockalingam, Raman V Kalyan.

Study search and selection: Julie Hogg, Josette Bettany-Saltikov, Hans-Rudolf Weiss, Razvan Taranu, Shreya Srinivas.

Methodological assessment: Victoria Whittaker, Hans-Rudolf Weiss, Razvan Taranu, Shreya Sriniva, Josette Bettany-Saltikov.

Acquisition/abstraction of data: Victoria Whittaker, Razvan Taranu, Shreya Sriniva.

Data analysis: Victoria Whittaker, Josette Bettany-Saltikov.

Interpretation of data: Victoria Whittaker, Nachiappan Chockalingam, Josette Bettany-Saltikov.

Drafting of the article: Josette Bettany-Saltikov, Hans-Rudolf Weiss, Razvan Taranu, Shreya Srinivas, Nachiappan Chockalingam, Raman V Kalyan.

Critical revision for important intellectual content: Josette Bettany-Saltikov, Hans-Rudolf Weiss, Razvan Taranu, Shreya Srinivas, Nachiappan Chockalingam, Raman V Kalyan.

Final approval of the version to be published: Josette Bettany-Saltikov, Hans-Rudolf Weiss, Razvan Taranu, Shreya Srinivas, Nachiappan Chockalingam, Julie Hogg, Raman V Kalyan, Victoria Whittaker.

Please note that some of these roles are still under negotiation and may change before the actual review is conducted.

Surgical versus non-surgical interventions in patients with adolescent idiopathic scoliosis (Protocol) 
DECLARATIONSOF INTEREST

Nothing to declare. 